\title{
THE EXPLORATION OF THE ORBIT *
}

\author{
BY \\ W. Hedley Summerskill \\ From the Portsmouth and Southern Counties Eye and Ear Hospital, \\ and St. Mary's Hospital, Portsmouth.
}

THE PRESENCE of space-taking lesions in the orbit can be diagnosed with a fair degree of confidence, but difficulty frequently arises in determining the nature, the size, and the exact position of such tumours. Those'near the outlet of the orbit and those outside the periorbita do not usually present this difficulty; but when surgery is required for deeper tumours in the intermediate space and in the muscle cone, the operation is to some extent an exploratory procedure. It should, therefore, be planned so that it can be adapted to deal with any condition that is found. It is usual to employ for these cases a sub-periorbital approach, following either a Kronlein osteoplastic resection or an incision down to the margin of the orbit. The transconjunctival route, advocated by Reese and others, is rarely employed, despite certain advantages, as it is generally assumed that it does not provide a satisfactory exposure of the larger tumours. In order to determine the validity of this assumption and define the limitations of this form of exploration of the orbit, the technique described below was employed for eight consecutive cases, seven of which were found to have intra-orbital tumours.

The following modification of the transconjunctival approach is designed to provide access to all parts of the orbital cavity by displacing the eye after detaching such fascia and muscles as necessary. A free lateral canthotomy is carried out down to the orbital margin and the lateral palpebral ligament and the palpebral fascia are freed from their attachment to the periosteum. The horizontal incision in the conjunctiva is extended upwards or downwards, depending on the estimated position of the tumour, keeping a few millimetres to the bulbar side of the fornix. If the inner side of the orbit is to be explored, this incision is continued round to the internal rectus. Digital palpation through the incision now enables the contents of the muscle cone and the intermediate space to be examined.

If the tumour is palpated in the cone, the external rectus is cut between sutures, the eye fully adducted, and the contents of the muscle cone exposed. If the tumour is found to be in the intermediate space, the conjunctival incision is extended as required. The tumour is localized by palpation and mobilized by blunt

- Received for publication December 9, 1949. 
dissection. Where necessary more room is obtained by section of the recti and displacement of the eye.

Careful adjustment of the muscles and suture of the conjunctiva and skin are required when the removal of the tumour is completed to secure a satisfactory post-operative position of the eye and to preserve the conjunctival cul-de-sac. Two months after operation it may be necessary to correct any residual deviation.

When, as in Cases 2 and 3, the tumour is found attached to the eye, this operation allows enucleation to be carried out without the disturbance of periorbita and intra-orbital fat created by the subperiosteal approaches, thereby leaving a better socket and involving less surgical trauma.

In only one case (No. 8) was resection of bone found necessary. This very vascular tumour extended into the apex of the orbit, and it was considered desirable to secure more room to control the entering vessels. In such rare cases displacement of the wall of the orbit can be carried out by extending the skin incision by two cuts along the orbital margin, making it into a horizontal $\mathrm{Y}$ shape. An osteoplastic resection can then be carried out, giving a better exposure than the classical Kronlein method, which leaves a bridge of skin and tissue between the displaced bone and the eye.

When, as in Cases 4 and 7, the exploration shows an exenteration to be advisable, this can be carried out more satisfactorily than by the Kronlein or other sub-periosteal approaches, for the periorbita has not been disturbed. The instruments are changed, an incision is made peripheral to the original incision, and the contents of the orbit are removed in an intact sac of periosteum.

Case 1.-This man gave a history of a sensation of pressure on the left eye and recurrent attacks of oedema of the upper lid which had persisted for over

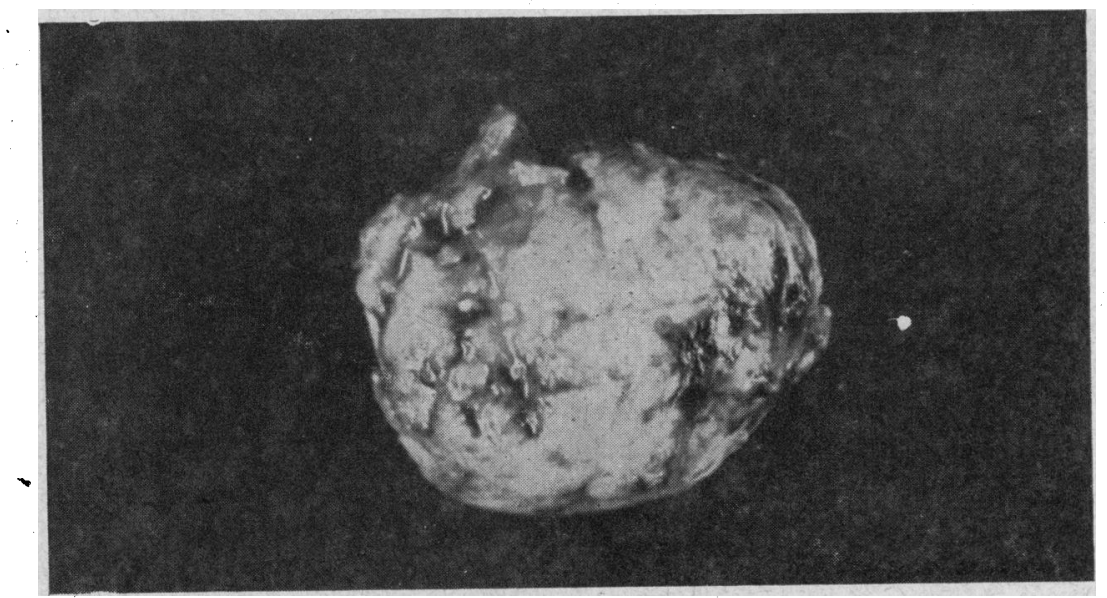

FIG. 1.-Case 1, mixed tumour of the lacrimal gland. $(1.2 \times 1.5 \times 1.5 \mathrm{~cm}$. 
three years. The left vision was $6 / 5$, no diplopia or limitation of movement could be detected, and clinical investigations revealed no abnormality. Some six months later after another attack of oedema, it was possible to palpate, beneath the rim of the orbit, what was assumed to be a tumour of the lacrimal gland. The gland was exposed by the technique under review and the tumour removed.

Extract from Pathological Report.-A mixed-celled tumour of the lacrimal gland, $1.2 \times 1.5 \times 1.5 \mathrm{~cm}$. Completely removed.

Such a tumour could have been removed through an incision below the brow, but before exposure there was no assurance that one was dealing with a relatively benign encapsulated mass. Had this not been the case, the approach used would have enabled other tumours in this area with possible extensions deep into the orbit to have been treated. Moreover, the transconjunctival approach leaves a smaller scar, and a few weeks after the operation there was little evidence of an operation having been performed.

Case 2.-A history of proptosis of the left eye for nine years was given. The eye had no perception of light; pupil inactive to direct, active to consensual stimulation, movements equally limited in all directions with a proptosis straight forwards. There was papilloedema, and a diagnosis of optic neuro-fibroma appeared probable. Clinical investigations revealed nothing abnormal except a strongly positive Wassermann reaction. Treatment for the latter carried out for eighteen months led to no improvement in the proptosis, which was, in fact, considered to have increased. Exploration of the orbit was therefore carried out. The method under review gave perfect access to contents of the muscle cone after cutting the external rectus, and a tumour of the optic nerve was exposed. It was found to merge with the sclera anteriorly, making it impossible to separate the eye. The conjunctival incision was, therefore, continued around the eye and an enucleation carried out, cutting the nerve well back behind the growth.

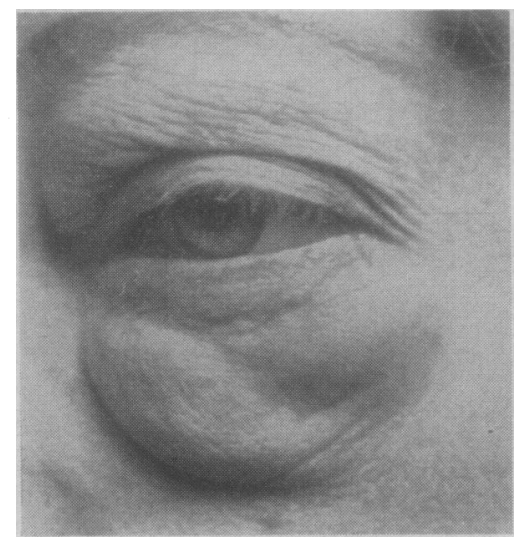

FIG. 2.-Case 2, showing left proptosis.

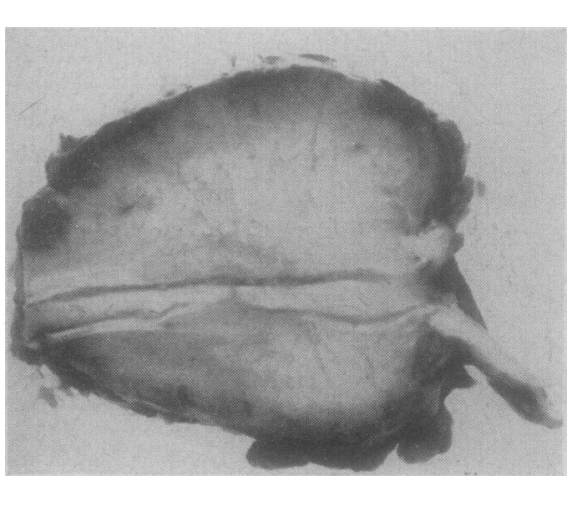

FIG. 3.-Case 2, meningioma of the optic nerve $(2.5 \times 2.8 \times 2.2 \mathrm{~cm}$. $)$

Extract from Pathological Report.-Situated around the optic nerve is a domeshaped tumour of $2.5 \times 2.8 \times 2.2 \mathrm{~cm}$., its base in close relationship to the sclera. Meningioma of the optic nerve. 
Conditions such as in this and the next case, which involve sacrificing the eye, can be dealt with as a simple enucleation, and surgery of the bony orbital wall is redundant. The approach under review enables the position to be recognized and the enucleation to be carried out without preliminary damage to the bone, periorbita, and intra-orbital fat. This technique, therefore, gives less operative trauma, a much more satisfactory socket for a prosthesis, less disfigurement, and a shorter convalescence than will result from a preliminary osteo-plastic resection.

Case 3.-A history of an operation for detached retina in the left eye nine years ago followed by gradual loss of sight was given. The previous day swelling and pain had commenced. On examination, a left proptosis and oedema of the lids were found to be present. There was no perception of light, the tension was raised, the anterior chamber shallow and the lens pushed forward and opaque. The sudden onset was suggestive of a haemorrhage but the history made it necessary to consider the possible presence of a choroidal sarcoma. Clinical investigations revealed no relevant abnormalities and the orbit was exposed by the method under review. When the muscle cone was opened a large posterior staphyloma was found, suggestive of an intra-ocular tumour commencing to perforate the sclera. The contents of the muscle cone were examined and a small nodule found lying on the optic nerve, otherwise the orbital contents appeared unaffected. The question was whether these findings justified continuing the operation as an exenteration of the orbit, as in the next case, or whether a free removal of the eye, optic nerve, and contents of the muscle cone would meet the case. The latter course was adopted.

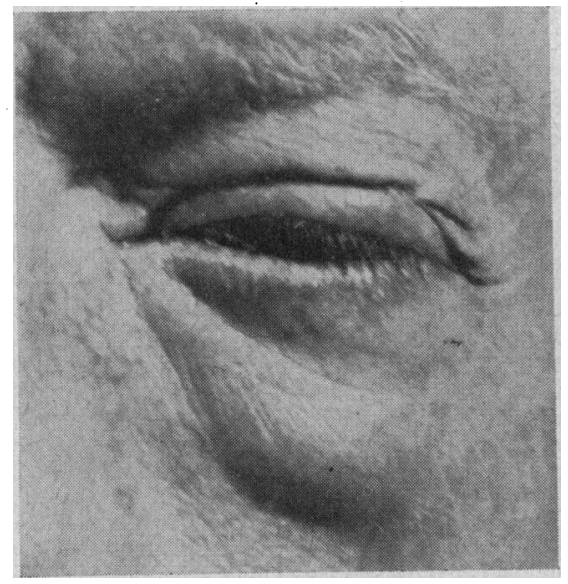

FIG. 4.-Case 3, showing left proptosis.

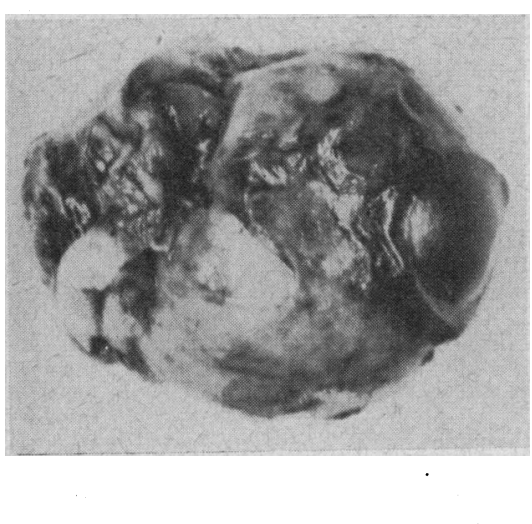

FIG. 5.-Case 3, malignant melanoma of the choroid. Extra-ocular extension.

Extract from Pathological Report.-Situated in the posterior half of the globe there is a necrotic, spindle-celled, heavily pigmented, malignant melanoma of the choroid. The growth has invaded and penetrated the sclera forming a nodule externally. 
The approach used enabled a thorough study of the contents of the muscle cone to be undertaken without the additional, and in such cases unnecessary trauma, involved by a bone resection. When a new growth suspected of being malignant is discovered, this technique confines the preliminary surgery to within the periorbita and eliminates the risk of implanting tumour cells outside this membrane. If exenteration is found to be indicated it can, therefore, be carried out with the benefit of an intact periorbita. Alternatively, if more conservative surgery and postoperative radio-therapy is considered adequate, a more presentable socket will result than that following a preliminary exploration by the Kronlein-Wagner technique.

Case 4.- One year previously had been under treatment for malignant cervical glands on the right side, which subsided with radiotherapy. No primary growth was found. Some right proptosis, oedema of the lids, and pain had recently developed, vision had fallen to perception of light, and papilloedema was present. Clinical investigations revealed nothing significant. The technique under review

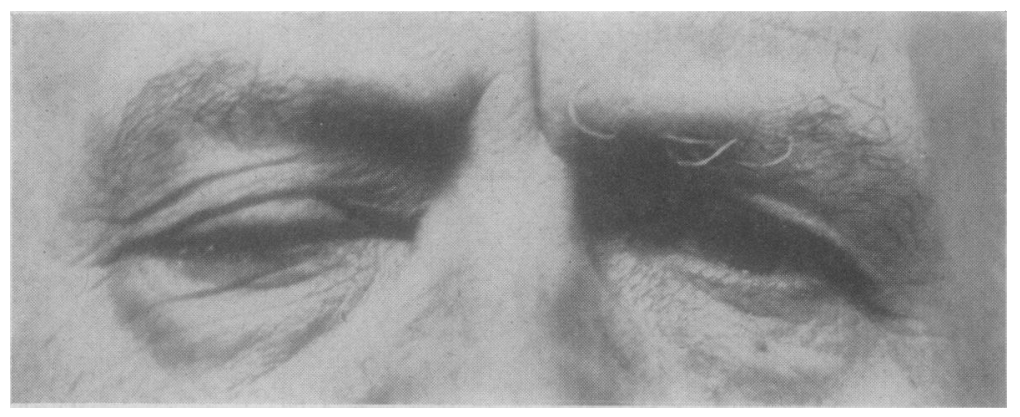

likg. 6.-Case 4, showing right proptosis.

was used for exploratory purposes and the apex and posterior third of the orbit were found to be filled by new growth. The instruments in use were discarded and an exenteration of the orbit leaving the lids was carried out through a conjunctival incision peripheral to the one used for the exploration.

Extract from Pathological Report. - Lympho-epithelioma probably extending from the nasal cavity or naso-pharynx, anaplastic carcinoma.

The use of the Kronlein approach in such a case would involve an incision being made through the periorbita into a cavity containing a malignant growth. The sub-periosteal space is, therefore, liable to contamination and the virtue of an exenteration - the removal of the orbital contents in an intact sac of periosteum -is lost. The technique under review allows the exploration to be carried out within the confines of the periosteum and, if malignancy is found, the instruments can be changed and a more efficient exenteration can be performed than after the Kronlein approach. 


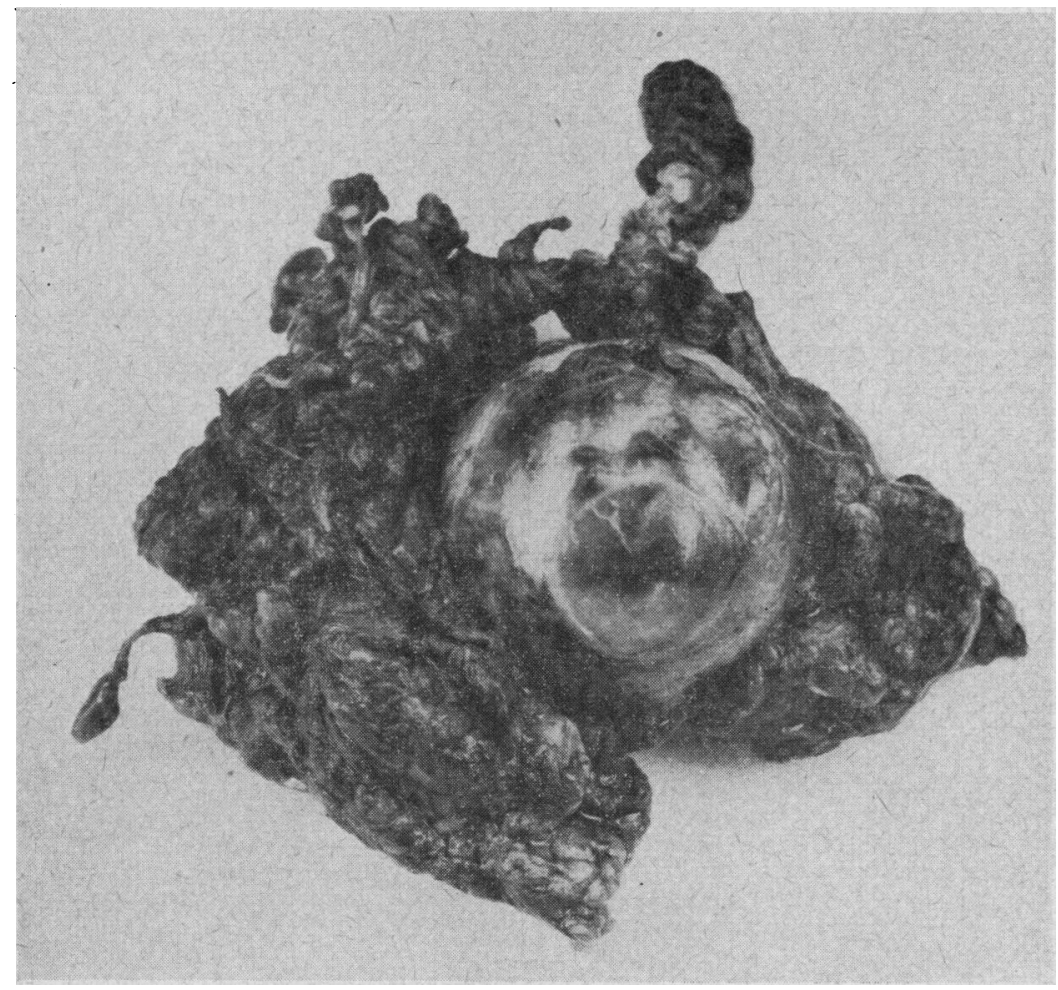

FIG. 7.-Case 4, anaplastic carcinoma of the nasal cavity. Orbital invasion.

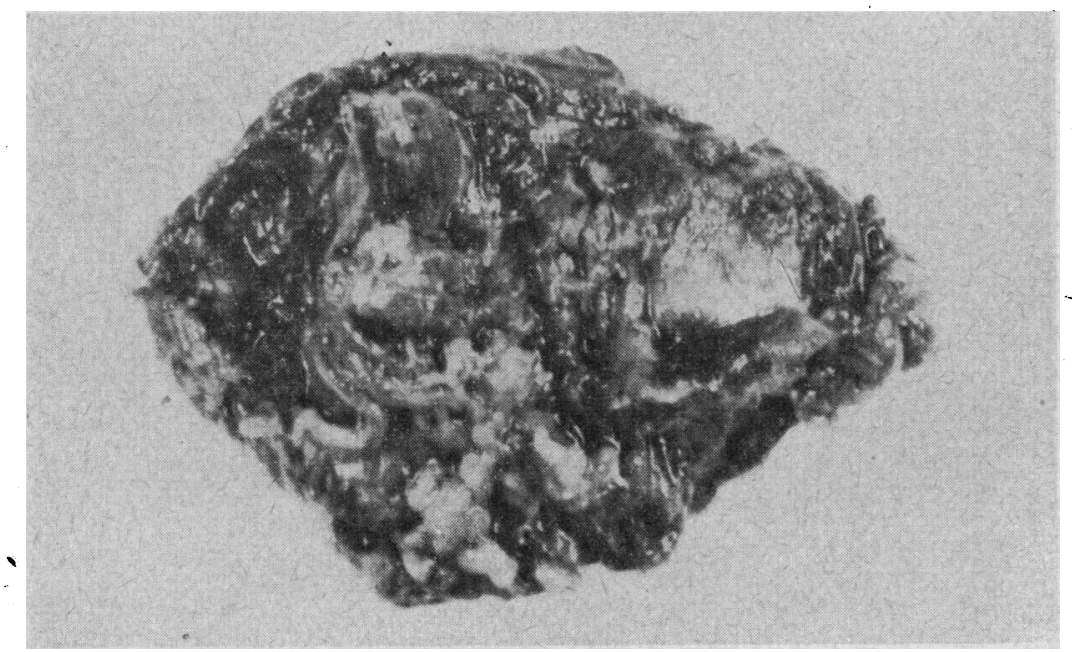

Fig. 8.-Case 5, pseudo-tumour of the orbit. ? Tuberculoma. $(4.3 \times 3.1 \times 3 \mathrm{~cm}$.) 
Case 5.-A history was given of discomfort around the left eye and diplopia of recent origin. An ill-defined irregularity within the medial extremity of the left inferior orbital margin was palpable. Clinical investigation revealed no material abnormalities. The tumour and the limitation of movement rapidly increased. Exploration by the method under review exposed a large tumour occupying the medial two-thirds of the floor of the orbit, extending behind the eye and enclosing the inferior rectus and oblique, the medial rectus, and the optic nerve. The exposure provided by the transconjunctival approach permitted a satisfactory dissection and complete removal, without removal of the eye (Fig'. 8).

Extract from Pathological Report.-The tumour consists of dense fibrous tissue infiltrated with chronic inflammatory cells. Throughout there are aggregations of lymphocytes around small blood vessels and an occasional giant cell may be seen.

This exceptionally large tumour, most of it in the medial half of the orbit, was mobilized and removed without difficulty by lateral canthotomy and a conjunctival incision. Resection of the lateral orbital wall would have given no more space and would have added to the severity of the operation and the post-operative disfigurement.

Case 6.- This patient had a seven-years' history of right proptosis and some limitation of movement. The condition had been stationary and was considered to be due to a partial third nerve paralysis resulting from a cerebral abscess. During the past year she had complained of discomfort and pressure on the right eye, and she considered that the proptosis was increasing. No objective evidence of this could be found, but owing to her insistence it was considered advisable to explore the orbit. This was carried out by the technique under review. The external and superior recti were cut and this provided a sufficiently satisfactory exposure to confirm the absence of any intra-orbital tumour. The opportunity was taken of shortening the superior rectus, and the final result was an improvement in the patient's appearance as the result of the exploratory operation instead of any disfigurement.

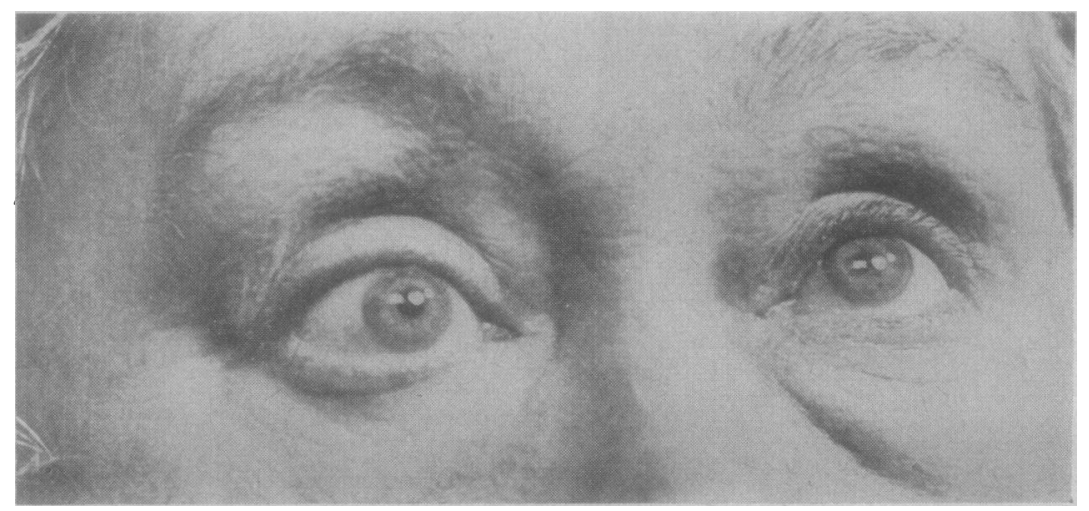

FIG 9.-Case 6, showing right proptosis.

The technique under review is particularly valuable when no tumour is believed to be present but confirmation of the opinion is considered desirable. Less hesitation need be fett in under- 
taking the exploration by a method involving so little surgical trauma and also giving the opportunity, as in this case, of improving the appearance of the patient by muscle surgery.

Case 7.- This patient had had both breasts removed five years previously owing to carcinoma and was reported to have multiple secondary deposits. Nodules could be palpated within the lower orbital margin consisting presumably of orbital metastases. In view of the hopeless prognosis, no surgery was at first considered desirable. But representations were made by her doctor for the orbital growth to be removed if possible, as she was being kept in ignorance of the recurrence of the disease and the gradually increasing proptosis and the discomfort were making her very unhappy. As her general health was still fairly good, it was considered under these circumstances that the removal of the orbital growth was justifiable. As the prognosis was hopeless and the object was only to give temporary comfort and to improve her appearance, a complete exenteration was undesirable. The technique under review was employed to study the extent of the new growth and it exposed a flat white plaque occupying the lateral half of the floor of the orbit. The tumour, the periorbita of the lateral two-thirds of the orbit, the eye, the superior, inferior and lateral recti, and the inferior oblique were removed. Sufficient conjunctiva was retained to cover the bare bone and a socket capable of retaining a prosthesis was obtained after three weeks. It was later reported that the patient survived for five months and that she was not bothered by pain in the orbit until too late in the illness to cause her much concern.

Extract from Pathological Report.-Metastatic carcinoma with much necrosis in places.

This partial exenteration was undertaken merely to give temporary symptomatic relief while avoiding gross disfigurement. This approach enabled the orbit to be examined and an estimate reached of the tissues to be conserved, more satisfactorily than by any other exploratory technique.

Case 8.-A history was given of a gradually increasing left proptosis for seventeen years. The left eye was so proptosed as to be nearly dislocated and the

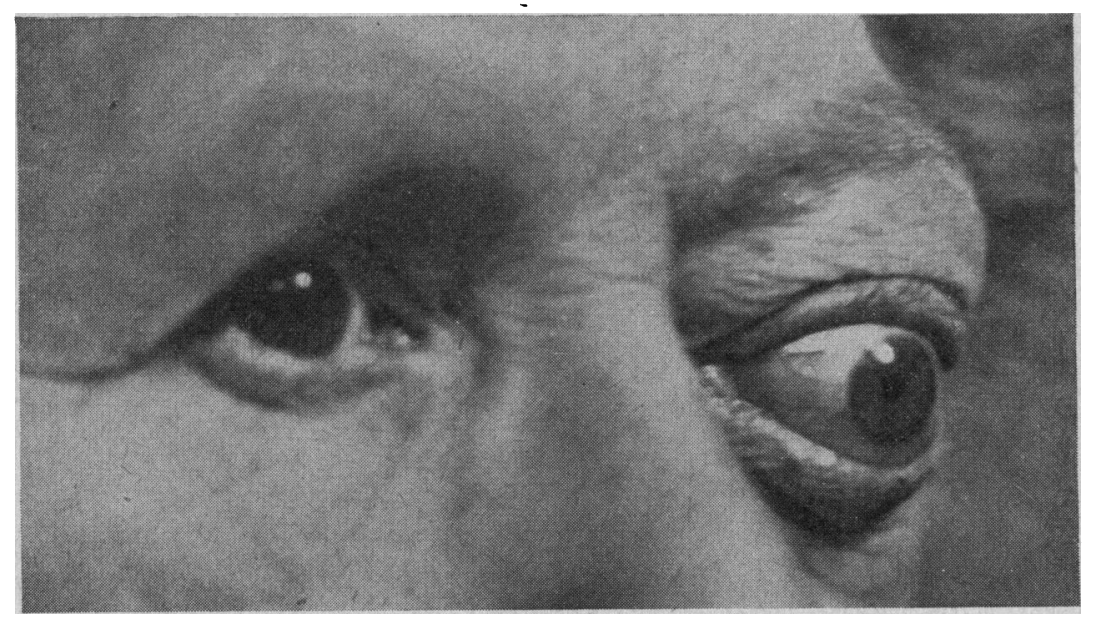

FIG. 10.-Case 8, showing left proptosis. 
conjunctiva and lids were oedematous. Investigation revealed no relevant abnormalities. Exploration was undertaken by the method under review. A cavernous haemangioma was found to occupy the whole of the orbit except the infero-nasal quadrant. The orbital fat was absorbed and replaced by new growth which extended right to the apex. 'The exposure given by the technique under consideration allowed the tumour to be defined and dissected free from the eye and orbital walls, after resection of the external and inferior recti. In view of the danger of haemorrhage from blind dissection at the apex of the orbit which was completely occupied by growth, it was considered desirable to get better access to the posterior aspect of the tumour by displacing the lateral orbital wall. 'The technique in use enabled this to be conveniently carried out, and as this still did not give sufficient exposure, the bone was temporarily removed. 'The tumour was then removed and the lateral wall replaced. 'The eye was retained.

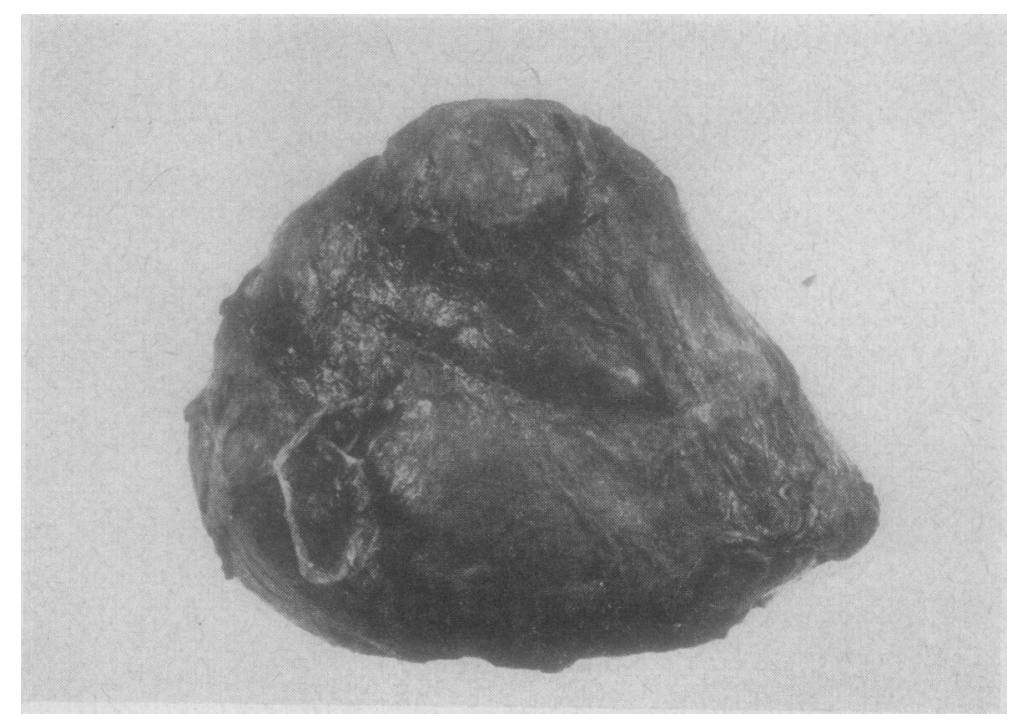

FIG. 11.-Case 8 , cavernous haemangioma. $(3.6 \times 2.7 \times 3 \mathrm{~cm}$.

Extract from Pathological Report.-Wedge-shaped, reddish tumour $3.6 \times 2.7 \times 3$ cm. On section it contains many minute spaces filled with blood. A cavernous haemangioma with evidence of old haemorrhages. There is no evidence of malignancy. The tumour appears to have been completely removed.

This was the only tumour in the serjes requiring bone resection, and then rather because of the necessity of controlling a potential haemorrhage than because of its size. It was an exceptionally large tumour occupying nearly all the retrobulbar space, and the fact that it could be mobilized without bone resection suggests that the exposure given by the method under review should be adequate for the majority of tumours. 


\section{Conclusions}

Unless the pre-operative investigations indicate the desirability of some specific form of orbitotomy, it appears that a transconjunctival approach combined with a free canthotomy provides a satisfactory method of exposing the orbit and dealing with any lesion found. It satisfies the three main requirements of such an operation.

(1) Adequate access is provided to every part of the orbit. Preoperative investigations may localize the main mass of a tumour, but an operation giving an adequate exposure of any offshoots is essential, for incomplete removal of some benign tumours predisposes to a recurrence, which is occasionally malignant. An example was Case 5, who appeared to have only a small tumour lying on the medial part of the floor of the orbit near the opening. But at operation this was found to extend well backwards behind the eye, to embrace the optic nerve, and to measure $4.3 \times 3.1 \times 3 \mathrm{~cm}$. Despite the retrobulbar extension, it was completely removed by the transconjunctival approach; the Kronlein method would have added to the operative trauma and given no better access, while the approach through an incision in the lower lid would have provided a totally inadequate exposure.

(2) The object of the operation is secured with minimal disfigurement and surgical trauma. Any orbit containing a tumour of doubtful pathology should be explored, but if the operation involves much operative risk or facial disfigurement there is a temptation to temporize in some cases. An example was Case 3, who suddenly developed a left proptosis, pain, and oedema of the lids, with an increased ocular tension in an old blind eye. The clinical picture was not pathognomonic of malignant disease of the orbit and some delay might have been justifiable if this had involved a major procedure. Another example was Case 6, complaining of an increase of an old-standing proptosis and discomfort for which there was no objective evidence. One suspected that an anxiety state rather than a tumour was the cause of the symptoms and one would have hesitated to undertake any exploration which involved any risk of disfigurement. As the technique described involves little trauma or post-operative deformity, exploration in such cases can be carried out immediately clinical investigations fail to identify the nature of the condition.

(3) The surgical approach should be one which does not jeopardize the efficient performance of any further procedure made necessary by the condition discovered. In this respect a transconjunctival approach to the orbit is preferable to other methods. Small encapsulated intra-orbital tumours can be removed with a minimum of trauma, and the conjunctival incision can be extended, 
and the muscles resected as required, to give access to every part of the orbit and to deal with any other condition. If bony resection be found necessary, it can be done by extending the skin incision. More important is the fact that with this approach no incision is made through the periorbita. As a result, if the exploration reveals malignant disease, the instruments can be changed and the contents of the orbit exenterated in an intact periosteal sac.

The author wishes to express his thanks to Dr. Charles Swan and Dr. Norman Ashton of the Institute of Ophthalmology, London, for their detailed pathological reports. 